\title{
The avian transcription factor c-Rel is induced and translocates into the nucleus of thymocytes undergoing apoptosis
}

\author{
Christelle Huguet ${ }^{1}$, Virginie Mattot ${ }^{1}$, Fatima Bouali ${ }^{1}$, \\ Dominique Stéhelin ${ }^{2}$, Bernard Vandenbunder ${ }^{1}$ and Corinne \\ Abbadie $^{1,3}$ \\ ${ }^{1}$ Regulation des processus invasifs, de l'angiogenèse et de l'apoptose, EP560 \\ CNRS/Institut Pasteur de Lille, Institut de Biologie de Lille, 1 rue du Professeur \\ Calmette, BP447, 59021 Lille, France \\ 2 Mécanismes du développement et de la cancérisation, UMR319 CNRS/Institut \\ Pasteur de Lille, Institut de Biologie de Lille, 1 rue du Professeur Calmette, \\ BP447, 59021 Lille, France \\ ${ }^{3}$ corresponding author: tel: 333 20871090, fax: 333 20871019, \\ email: cabbadie@infobiogen.fr
}

Received 24.2.97; revised 10.3.97; accepted 11.3.97

Edited by G. Melino

\begin{abstract}
This study investigates the involvement of the avian transcription factor c-Rel in thymocyte apoptosis occurring either in vivo or in organotypic culture. In vivo, only a few cortical thymocytes express the c-Rel protein. Their number, localization and morphology resemble that of apoptotic cells evidenced by TUNEL staining. In organotypic culture, the expression of c-Rel is induced in medullary thymocytes as apoptosis is triggered. This induction would be posttranscriptional since no increase in the c-rel gene expression is detected. Moreover, c-Rel translocates into the nucleus of medullary thymocytes during the time course of apoptosis. This translocation is preceded by a decrease in ikba expression, the gene which encodes the avian homologue of $I_{\kappa} B \alpha$. Altogether these results suggest that the protooncogene c-rel could take an active part in apoptosis of cortical thymocytes occurring in vivo during T-cell selection as well as in experimentally-induced apoptosis of medullary thymocytes.
\end{abstract}

Keywords: Rel/NF- $\kappa$ B; transcription factor; apoptosis; avian thymus

Abbreviations: NF- $\kappa \mathrm{B}$ : Nuclear factor $-\kappa \mathrm{B}$; TUNEL: TdTmediated dUTP nick end labelling; RHD: Rel homogy domain; AEC: 3-amino-9-ethylcarbazole

\section{Introduction}

Cell fate depends on informations addressed by the extracellular environment via the cell membrane and through various signalling cascades to the nucleus. In response, a wide array of genes are switched 'on' and 'off' leading to cell proliferation, differentiation or death. Switch of gene expression is driven by transcription factors which are usually classified in families, where individual members perform specific or similar tasks. Rel/NF $\kappa$ B transcription factors form one of these families; every member shares a highly conserved 300 amino acids N-terminal domain, the Rel Homology Domain (RHD), which contains sequences necessary for DNA binding, dimerization, nuclear localization and interaction with inhibitory proteins of the $\mathrm{I}_{\kappa} \mathrm{B}$ family. Rel/ $\mathrm{NF}-\kappa \mathrm{B}$ proteins associate as homo- or heterodimers and bind a specific DNA sequence, the $\kappa \mathrm{B}$ motif, in the promoter of their target genes. In most cell types, Rel/NF- $\kappa$ B dimers are found in an inactive form sequestered in the cytoplasm by an ankyrin-repeat protein of the $\mathrm{I}_{\kappa} \mathrm{B}$ family. Upon stimulation, the $\mathrm{I}_{\kappa} \mathrm{B}$ inhibitor is phosphorylated and degraded, thus allowing the transcription factor to enter the nucleus. The Rel/NF- $\kappa \mathrm{B}$ family can be subdivided into two classes based on differences in the $\mathrm{C}$-terminal part of the proteins. The first class includes c-Rel, RelA (p65) and RelB as well as the drosophila proteins Dorsal and Dif. These proteins contain in their C-terminal part a transactivating domain. The second class consists of p50 and p52, two proteins devoid of transactivating domain and proteolytically maturated from precursor proteins, p105 (or NFKB1) and p100 (or NFKB2) respectively. p50 or p52 do not generally activate transcription as homodimers, but they become potent transactivators when they heterodimerize with RelA or c-Rel. Interestingly, p105 and p100 precursors contain $\mathrm{I}_{\kappa} \mathrm{B}$-like ankyrin-repeats at their C-terminus and behave as inhibitory proteins by complexing and retaining Rel/NF- $\kappa \mathrm{B}$ factors in the cytoplasm. These ankyrin-repeats are eliminated by proteolytic maturation (for reviews see Miyamoto and Verma, 1995; Gilmore et al, 1996). Rel/NF- $\kappa$ B transcription factors are ubiquitously expressed, activated by a great variety of stimuli and able to regulate the expression of genes as different as genes encoding immunoreceptors, cytokines or cell-adhesion molecules (for review see Bauerle and Henkel, 1994). Altogether these data suggest that Rel/NF- $\kappa$ B transcription factors could be involved in numerous physiological processes. Investigation of Rel/NF$\kappa \mathrm{B}$ physiological functions began with the study of the protooncogene c-re/ which was the first Rel/NF- $\kappa \mathrm{B}$ gene cloned as the cellular homologue of the oncogene v-rel (for review see Huguet et al, 1994). Northern blot analysis has revealed that the expression of c-rel is restricted to hematopoietic organs such as the bursa of Fabricius, thymus, spleen and bonemarrow (Moore and Bose, 1989). However, in situ hybridization experiments performed in young embryos from 3 to 9.5 days of development have indicated that c-rel is ubiquitously expressed at low levels while high levels of transcripts were detected in cells exhibiting an apoptotic morphology (Abbadie et al, 1993). These expression patterns suggested that c-rel may function in hematopoiesis and apoptosis. To evaluate the 
causal link between the expression of c-rel and the occurrence of apoptosis, the protein was overexpressed in two different types of primary cells. Chick embryo fibroblasts overexpressing c-Rel acquired a transformed phenotype and an extended life span in culture. In contrast, bone marrow cells overexpressing $c$-Rel failed to grow in culture and underwent programmed cell death (Abbadie et al, 1993). The c-rel functions in mammals were investigated more recently by the description of its expression pattern in embryonic and adult mice and by the establishment of null mice. In contrast to chicken c-rel, mouse c-rel transcripts were detected late in the embryonic development and restricted to hematopoietic organs. This pattern was conserved in the post-natal period. The c-Rel protein was found in erythroid precursors of the fetal liver, in mature splenic B cells and in thymic epithelial cells (Carrasco et al, 1994). c-rel-deficient mice exhibited an impaired immunity attributable to defects in the activation of mature B and T-cells but surprisingly no developmental defect in the hematopoietic system. In addition, no change in the occurrence of apoptosis was noticed (Köntgen et al, 1995). Thus, whereas a clear in vivo function of mouse c-rel is now established in the specific immune response, the significance of c-rel expression during embryonic development of hematopoietic organs remains elusive. Moreover, discrepancies between mouse and chicken were evidenced concerning the expression and function of c-rel in apoptosis. This prompted us to further study the proto-oncogene c-rel in the avian system and to focus on its involvement in apoptosis. The thymus was chosen as model since T-cell maturation and selection include the elimination of numerous non-functional thymocytes by apoptosis (for reviews see von Boehmer, 1994; Nossal, 1994).

We describe here the expression of c-rel during normal thymocyte apoptosis occurring in vivo and after induction of massive apoptosis in thymus organ culture. Since the activity of Rel/NF- $\kappa$ B proteins depends on interactions with $\mathrm{I} \kappa \mathrm{B}$ proteins that determine their nuclear or cytoplasmic localization, we have described the subcellular distribution of the c-Rel protein during the process of apoptosis together with the expression of $i k b a$, the gene encoding the avian homologue of $\mathrm{I} \kappa \mathrm{B} \alpha$ (Davis et al, 1991; Kerr et al, 1991). Our results indicate that the c-Rel protein is expressed in thymocytes undergoing apoptosis through natural selection and moreover that c-Rel is induced and translocates into the nucleus of thymocytes during the time course of experimentally-induced apoptosis. This nuclear translocation of $\mathrm{c}-\mathrm{Rel}$ is preceded by a decrease in ikba expression.

\section{Results \\ c-Rel is expressed during thymocyte apoptosis occurring through natural selection}

To evaluate the involvement of c-rel in apoptosis of thymocytes, we have studied the expression of the c-Rel protein by immunocytochemistry with an immunopurified anticRel antibody during thymus development. Since no data was available about the occurrence of apoptosis in the avian thymus, we have first performed a study by TUNEL, a method
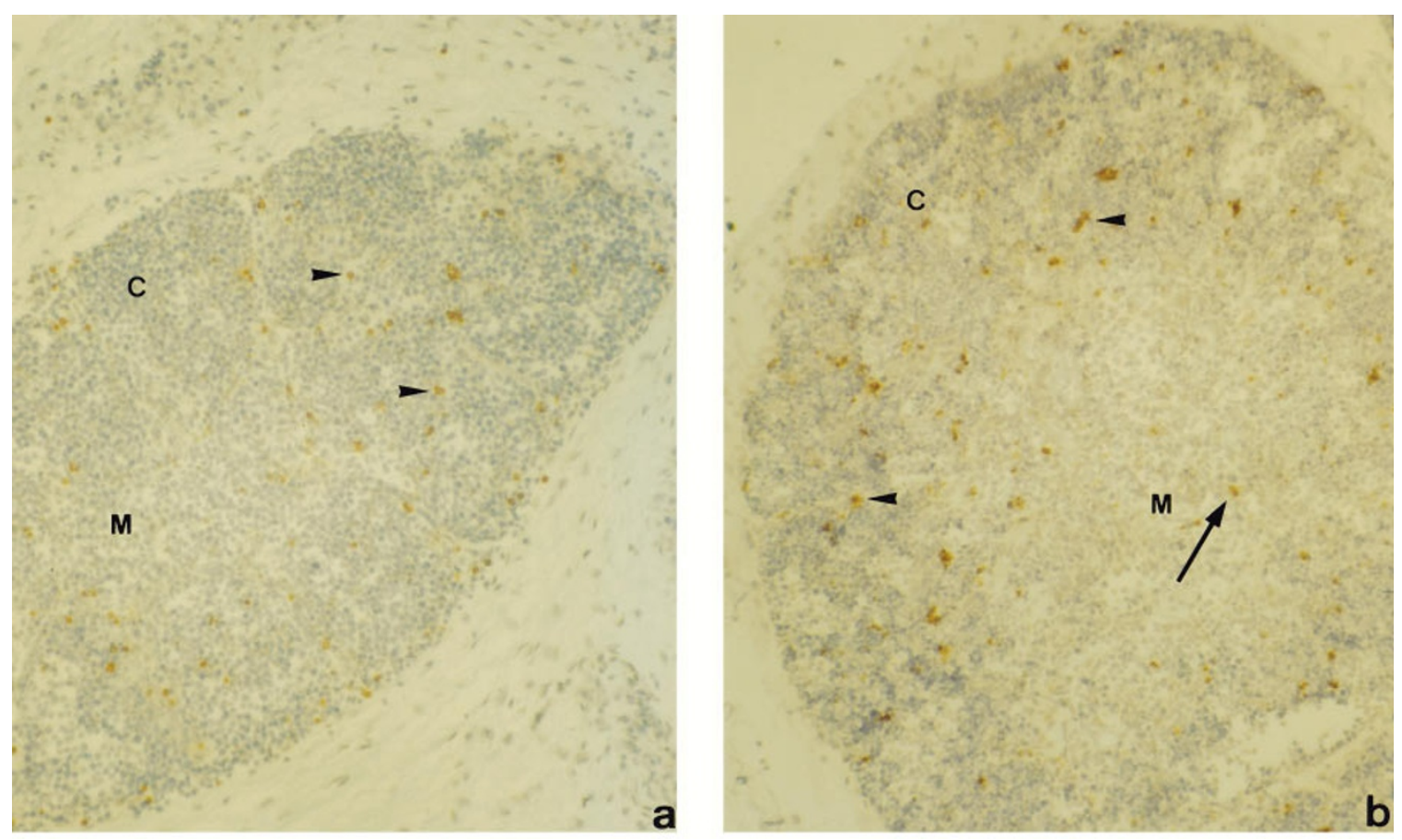

Figure 1 In situ detection of apoptotic cells during thymus development. E15 and P1 thymus sections were processed for TUNEL staining of apoptotic cells. Cortical and medullary areas are indicated as (C) and (M). Apoptotic cells are stained in brown compared to negative cells which are counterstained in blue by hematoxylin. At E15 (a) and P1 (b), apoptotic thymocytes are scattered in the cortex (arrowheads). Only a few cells are stained in the medulla (arrow). The number of apoptotic cells increases from E15 to P1 $\times 200$. 
which reveals apoptotic cells by detecting DNA strand breaks (Gavrieli et al, 1992). Experiments were done at four representative stages of the chick thymus development: at E9.5 when the thymic rudiment mainly contains thymocyte precursors; at E15 when cortical and medullary areas begin to separate indicating that thymocyte maturation is engaged and at $\mathrm{E} 19$ or $\mathrm{P} 1$ when the thymic structure is completed. At E9.5, almost no apoptotic cells are detected (data not shown). At E15, E19 and P1, apoptotic cells are mainly found in the cortex scattered among numerous healthy thymocytes whereas only a few apoptotic thymocytes are detected in the medulla (Figure 1). The major difference between E15, E19 and P1 is the amount of apoptotic thymocytes which increases from E15 to P1. It should be noticed that only a low number of apoptotic thymocytes are detected in situ in spite of the high number of thymocytes that are eliminated through natural selection (Egerton et al, 1990). This apparent paradox can be explained considering that apoptosis is a rapid phenomenon and that dead cells are efficiently engulfed. In view of these results, we have focused our analysis of the expression of c-Rel in the cortex of thymuses from E15 to P1. The vast majority of cortical thymocytes do not express c-Rel. However, a few c-Rel-expressing thymocytes can be detected scattered all over the cortex. They are generally isolated among negative thymocytes but they can also form clusters (Figure 2d and e). A few c-Rel-expressing thymocytes appear condensed or even fragmented. The cellular fragments are round and grouped by two or three or have an irregular and damaged morphology (Figure $2 f$ and $g$ ). TUNEL-positive cells also appear isolated or in clusters, condensed or fragmented in apoptotic bodies more or less damaged (Figure 2a, b and c). From these morphological similarities between c-Relexpressing cells and TUNEL-positive cells, we assumed that the cortical thymocytes that express c-Rel undergo apoptosis.
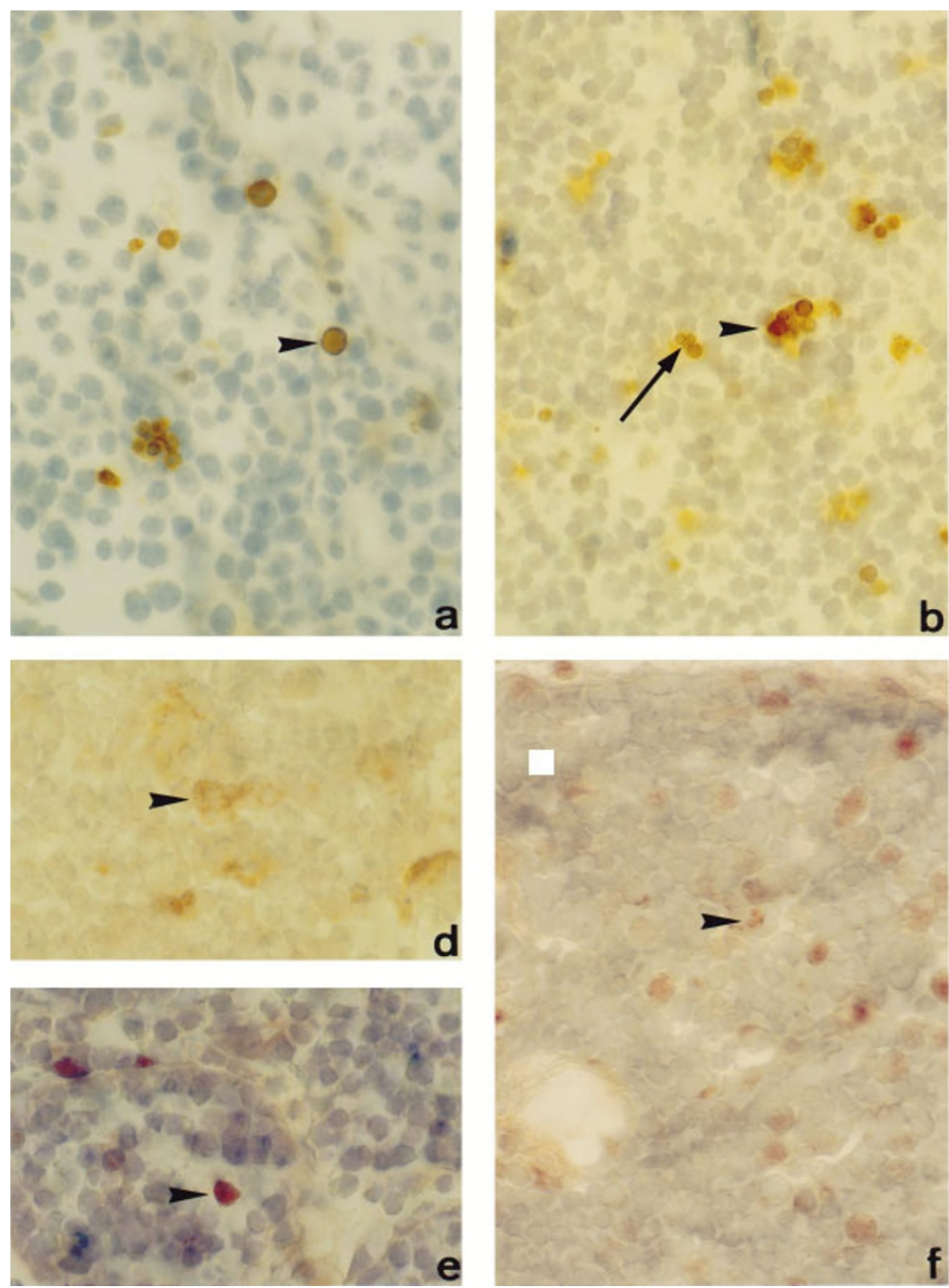
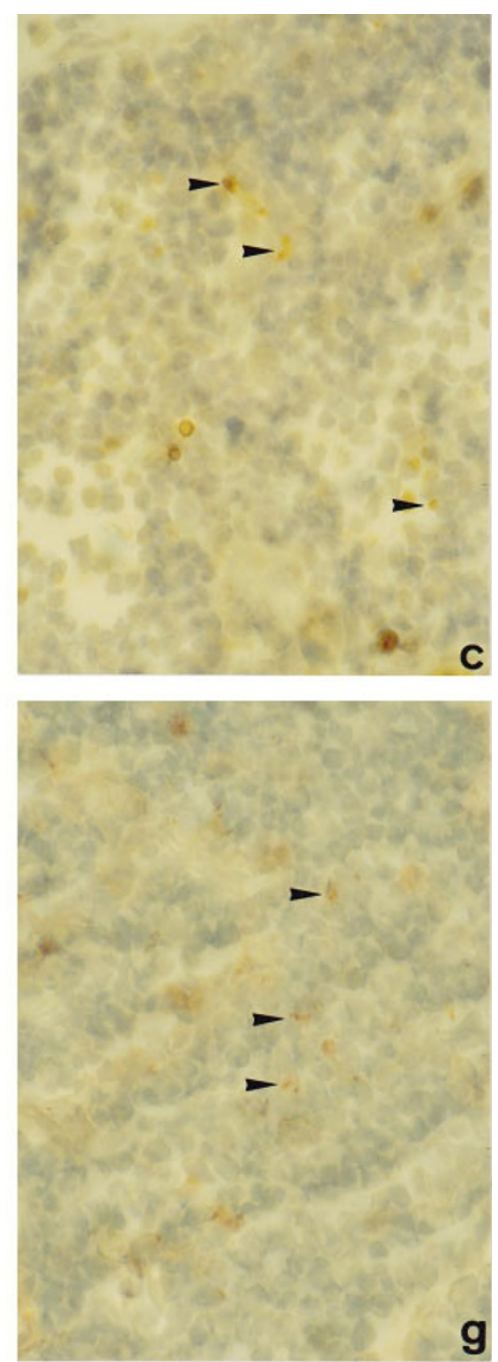

Figure 2 High magnifications of apoptotic and c-Rel-expressing thymocytes of the cortex. Thymus sections were processed for TUNEL staining (a, $\mathbf{b}$ and $\mathbf{c}$ ) or anti-c-Rel immunocytochemistry (d, e, $\mathbf{f}$ and $\mathbf{g}$ ). Apoptotic cells are stained in brown, $\mathbf{c}$-Rel-expressing-cells in red and negative cells in blue. TUNEL-positive thymocytes are either isolated (a, arrowhead) or in cluster (b, arrowhead). The TUNEL staining also detects apoptotic bodies (b, arrow) or cell debris (c) arrowheads). Similarly, c-Rel-expressing thymocytes appear in cluster (d, arrowhead) or as isolated cells (e, arrowhead). c-Rel is also detected in apoptotic bodieslike elements (f, arrowhead) and cell debris (g, arrowheads). $\times 600$. 


\section{c-Rel translocates into the nucleus of medullary thymocytes undergoing experimentally-induced apoptosis}

The scarceness of apoptotic thymocytes observable in a thymus section makes their in situ study difficult. To bypass this limitation and to check whether c-Rel is activated when thymocytes undergo apoptosis, we have developed a protocol for the induction of massive apoptosis inside the thymus. Dissection and organotypic culture of E19 or P1 thymuses in complete RPMI medium, at $40^{\circ} \mathrm{C}$, with $10 \% \mathrm{CO}_{2}$ create a stress sufficient to induce thymocyte apoptosis. TUNEL staining revealed that apoptosis occurs first in the medulla after $2 \mathrm{~h}$ in culture. The number of TUNEL-positive thymocytes increases in the course of the culture and at $4 \mathrm{~h}$ the majority of medullary thymocytes are apoptotic (Figure $3 \mathrm{~b}$, and d). Interestingly, $4 \mathrm{~h}$ in culture do not affect the level of apoptosis occurring in the cortex. However, when the culture is maintained over $24 \mathrm{~h}$, cortical thymocytes also undergo apoptosis (data not shown). Stromal cells remain unaffected by the culture until $5 \mathrm{~h}$ (data not shown). According to these results, we have studied the expression of c-Rel in medullary thymocytes of thymuses maintained in organotypic culture up to $4 \mathrm{~h}$. Thymuses were fixed at different times of culture and serial sections were processed either for immunocytochemistry or TUNEL staining. Experiments were done both with
E19 and P1 thymuses and gave identical results. In control thymuses, medullary thymocytes do not express c-Rel and are TUNEL-negative (Figure $3 a$ and $b$ ). Beside thymocytes, some medullary cells express c-Rel; they were previously identified as dendritic cells, epithelial cells and macrophages (unpublished observation). After $1 \mathrm{~h}$ in culture, no change appears either in the expression of c-Rel or in the occurrence of apoptosis (data not shown). After $2 \mathrm{~h}$ in culture, c-Rel is faintly detected in numerous medullary thymocytes which still display a healthy morphology (data not shown). The TUNEL staining reveals only a few positive cells, indicating that most thymocytes are still healthy but that apoptosis has already begun (data not shown). After $3 \mathrm{~h}$ in culture, a great number of medullary thymocytes are TUNEL-positive. Numerous thymocytes express c-Rel and among them some are highly stained (data not shown). After $4 \mathrm{~h}$ in culture, almost all medullary thymocytes are apoptotic; they are highly positive in immunocytochemistry (Figure $3 c$ and $d$ ). These results indicate that, in thymocytes, c-Rel expression is induced when apoptosis is induced.

To evaluate the activation of c-Rel during the process of apoptosis, we have determined its subcellular localization by observing the sections at high magnification. From 2 to $4 \mathrm{~h}$ in culture, the c-Rel protein is generally localized in the cytoplasm of thymocytes (Figure 4a). However, at 3 and $4 \mathrm{~h}$ in culture, several thymocytes display a staining of both
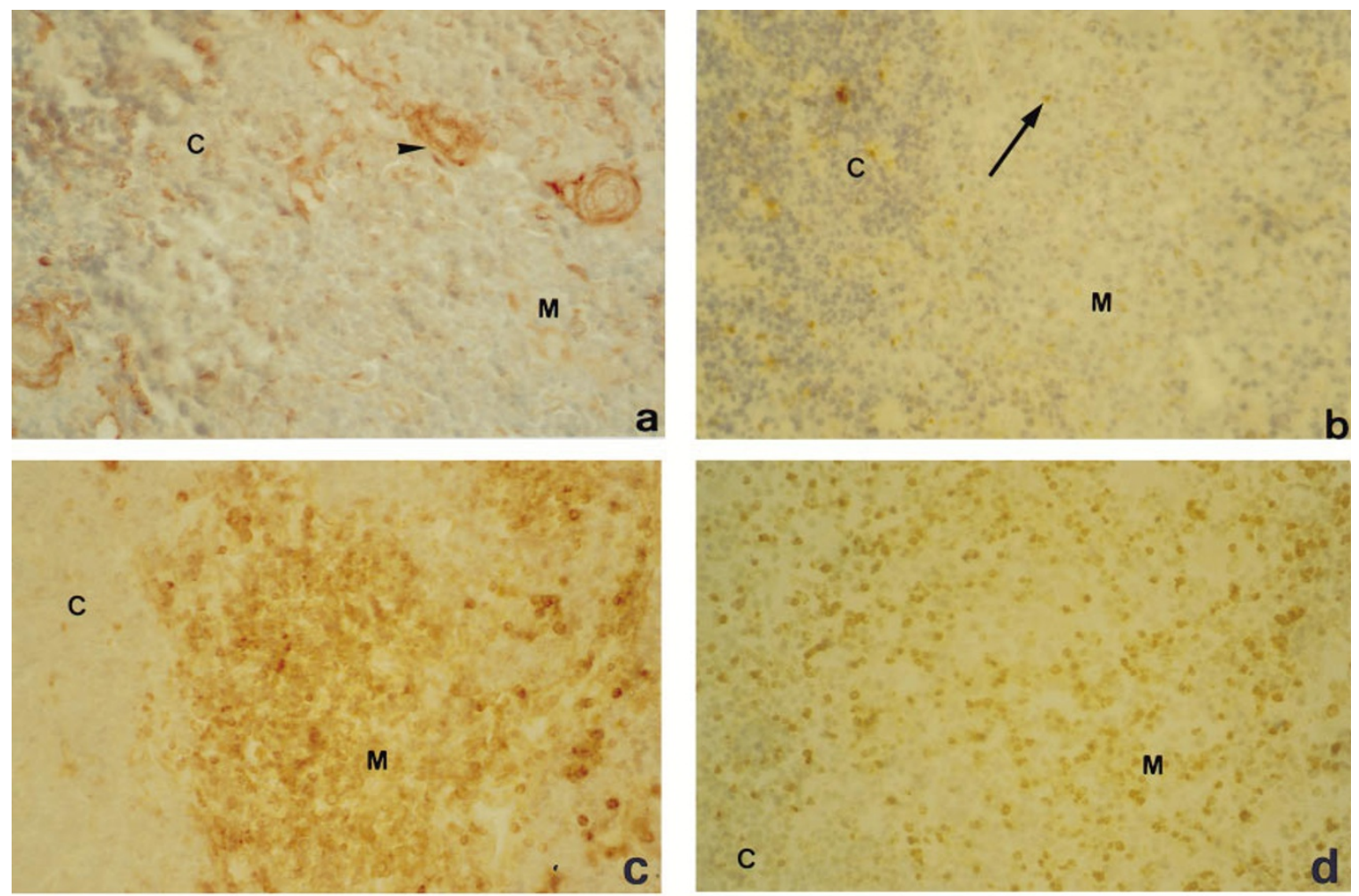

Figure 3 Detection of $\mathbf{c}-$ Rel expression after induction of apoptosis in thymus organ culture. P1 thymus sections were processed either for TUNEL staining (b and d) or anti-c-Rel immunocytochemistry ( $\mathbf{a}$ and $\mathbf{c}$ ) at different times of culture. Cortical and medullary areas are indicated as (C) and (M). Apoptotic cells are stained in brown, c-Rel-expressing-cels in red and negative cells in blue. In control thymuses (a and $\mathbf{b}$ ), cortical and medullary thymocytes do not express c-Rel in contrast to stromal cells that are highly positive (a, arrowhead); only very few medullary thymocytes are TUNEL positive (b, arrow). After $4 \mathrm{~h}$ in culture (c and $\mathbf{d})$, a majority of medullary thymocytes are highly stained in immunocytochemistry (c) and almost all medullary thymocytes are apoptotic as revealed by their TUNEL staining (d). $\times 250$ 
the cytoplasm and the nucleus, with however a higher staining of the cytoplasm (Figure $4 \mathrm{~b}$ and e). c-Rel is also detected in condensed thymocytes and in apoptotic bodies with an homogenous distribution, suggesting a localization in both the condensed cytoplasm and nucleus (Figure 4d). Therefore, c-Rel could be activated to translocate into the nucleus during apoptosis. To better address this point, we have performed Western blot analysis on nuclear and cytoplasmic extracts of thymus prepared at different times of culture. In control thymuses and until $2 \mathrm{~h}$ in culture, the $\mathrm{c}-\mathrm{Rel}$ protein is mainly found in the cytoplasmic fraction; very few amounts of protein are detected in nuclear extracts. At 3, 4 and $5 \mathrm{~h}$ in culture, c-Rel amounts in the nuclear fraction are clearly higher than in control thymuses (Figure 5) indicating that $\mathrm{c}-\mathrm{Rel}$ has translocated into the nucleus from $3 \mathrm{~h}$ of culture onwards. Taken together, immunocytochemistry and Western blot experiments show that $\mathrm{c}-\mathrm{Rel}$ is induced and activated to translocate into the nucleus during thymocyte apoptosis.

\section{The ikba gene expression decreases in thymocytes during apoptosis}

In order to investigate the mechanism of c-Rel induction and nuclear translocation during experimentally-induced apoptosis, we have examined the expression of the c-rel and ikba genes by in situ hybridization. ikba encodes the avian homologue of $\mathrm{I} \kappa \mathrm{B} \alpha$ which was shown to be able to sequester the avian c-Rel protein in the cytoplasm and to inhibit its binding to DNA (Diehl et al, 1993; Kerr et al, 1991; Davis et al,
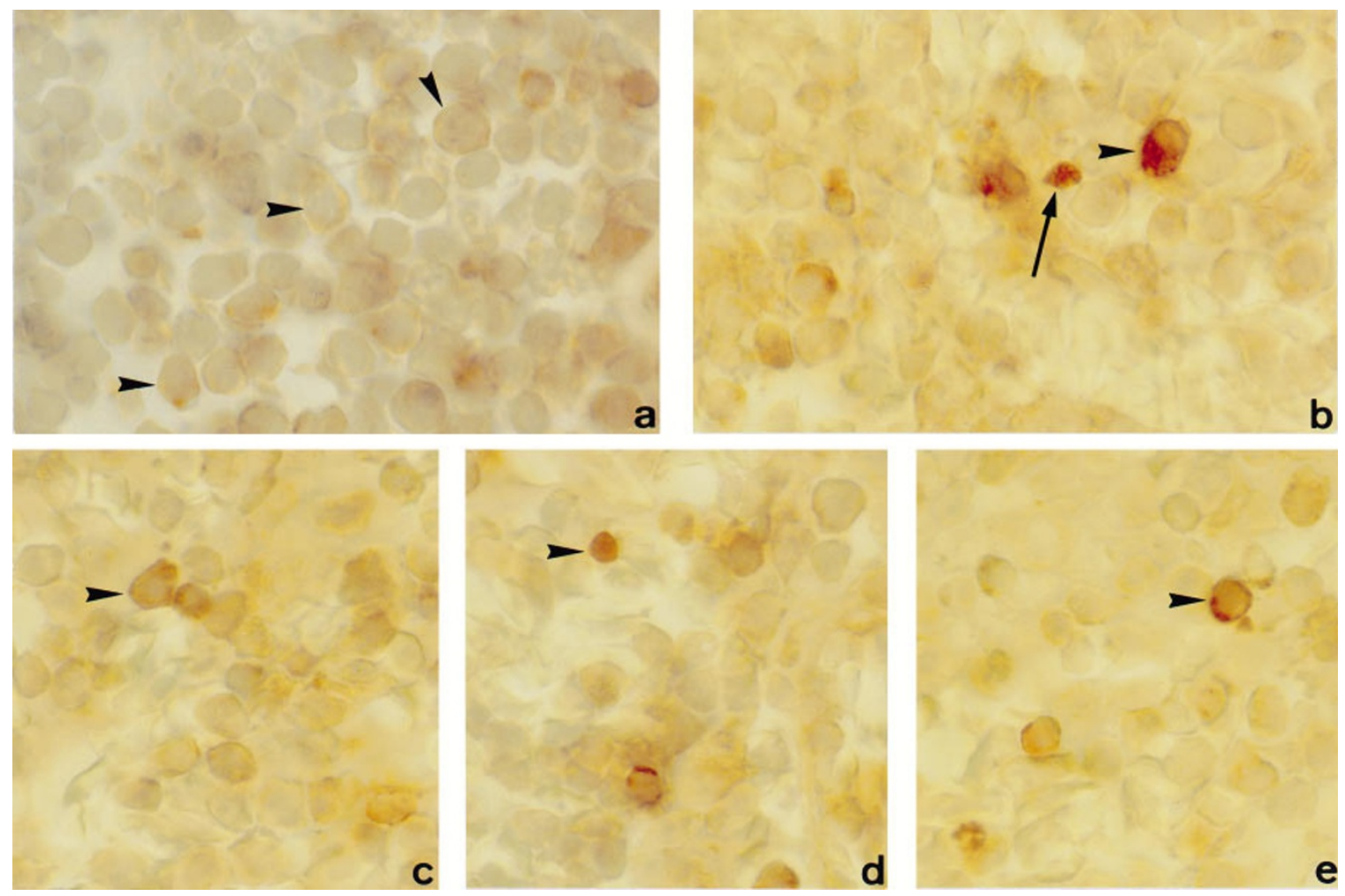

Figure 4 Subcellular localization of c-Rel during apoptosis of medullary thymocytes. P1 thymus sections were processed for anti-c-Rel immunocytochemistry and observed at high magnification. c-Rel is faintly detected in the cytoplasm of thymocytes after $2 \mathrm{~h}$ in culture (a, arrowheads). After $3 \mathrm{~h}$ in culture, some medullary thymocytes are highly stained either in the cytoplasm (c, arrowhead) or both in the cytoplasm and the nucleus, although less intensely in the latter (b and $\mathbf{e}$, arrowheads). c-Rel is also highly expressed in condensed thymocytes (d, arrowhead) and in apoptotic bodies (b, arrow). $\times 1250$.

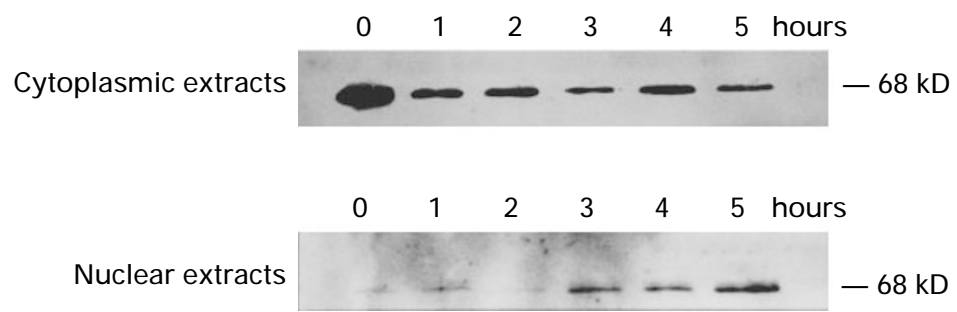

Figure 5 Western-blot analysis of cytoplasmic and nuclear extracts from E19 thymuses in organotypic culture. The anti-c-Rel antibody detects a protein at $68 \mathrm{kD}$, the molecular mass of the avian c-Rel protein (Capobianco et al, 1990). In thymus control and until $2 \mathrm{~h}$ in culture, the c-Rel protein is mainly found in cytoplasmic extracts: only few amounts are detected in nuclear extracts. After $3 \mathrm{~h}$ of culture, $\mathrm{c}$-Rel amounts increase in nuclear extracts. 
1991). Experiments were done with $\mathrm{E} 19$ and $\mathrm{P} 1$ thymuses and gave similar results. Serial sections were used for immunocytochemistry, TUNEL staining and in situ hybridization experiments and thus any changes in either gene expression, protein expression or occurrence of apoptosis were directly correlable. In control thymuses, c-relmRNAs are detected in both the cortex and the medulla (Figure 6a and c) with a higher level in medullary stromal cells (Huguet et al, 1997). In contrast, ikba is highly expressed in the medulla and more faintly in the cortex (Figure 6e and g). Inside the medulla, stromal cells give the most intense signal but it has to be noticed that thymocytes also highly express ikba (Huguet et al, 1997). After $1 \mathrm{~h}$ in culture, no change is observed in the expression of the c-rel and $i k b a$ genes. After $2 \mathrm{~h}$ in culture, crel mRNA expression remains stable (Figure $6 b$ and $d$ ) although, as described above, the c-Rel protein expression is already induced. In contrast, ikba expression decreases in thymocytes whereas it remains unchanged in stromal cells (Figure $6 \mathrm{f}$ and $\mathrm{h}$ ). It has to be reminded that at this stage the cRel protein is mainly localized in the cytoplasm of thymocytes and that its nuclear translocation is evidenced especially from $3 \mathrm{~h}$ in culture. Therefore, the decrease in ikba expression precedes and could account for the translocation of c-Rel into the nucleus of thymocytes undergoing apoptosis. After 3 and $4 \mathrm{~h}$ in culture, ikba expression still decreases but c-rel expression decreases too (data not shown). According to the high number of apoptotic bodies in the medulla at 3 and $4 \mathrm{~h}$ of culture, we estimated that decreases in gene expression are not due to specific mechanisms of regulation but rather to a too far engagement of the cells in the death process.

\section{Discussion}

Previous studies have shown that the mouse proto-oncogene c-rel is involved at two different levels in T-cell development: in mature T-cell activation and in T-cell education via an indirect role in thymic epithelial cells (Carrasco et al, 1994; Köntgen et al, 1995). No relationship between the expression of mouse crel and apoptosis could be evidenced in these experiments, thus contrasting with results obtained in the avian system where c-rel and its viral counterpart v-rel have been involved in apoptosis of hematopoietic cells as well as of cells of different origins during embryonic development (Neiman et al, 1991; White et al, 1995; White and Gilmore, 1996; Abbadie et al, 1993). In this study, we have investigated the involvement of the avian proto-oncogene c-rel in thymocyte apoptosis.

\section{c-Rel is induced in apoptotic thymocytes}

Considering the high amounts of c-rel mRNAs described in apoptotic cells in the avian embryo (Abbadie et al, 1993), we expected such an expression in the thymus cortex where a majority of thymocytes undergo apoptosis (Egerton et al, 1990). Surprisingly, only low levels of c-rel transcripts were detected in cortical thymocytes, slightly higher levels in medullary thymocytes and the highest levels were found in non-lymphoid cells (Huguet et al, 1997). Since apoptotic thymocytes are buried among numerous healthy cortical thymocytes, it was not possible to identify them in the in situ hybridization experiments and therefore to evaluate their level of c-rel expression. By contrast, we were able to show by immunocytochemistry that the c-Rel protein is only expressed in a few isolated cortical thymocytes that could be apoptotic since their number, localization and morphology resemble that of TUNEL-positive cells. This result suggesting that c-Rel could be induced during apoptosis, we have developed an assay to test whether c-Rel is induced when apoptosis is triggered experimentally. The organ culture protocol used for mouse thymus has been adapted to the very young E10 chick thymus (Davidson et al, 1992). When we applied this technique to fully developed E19 or P1 thymuses, medullary thymocytes underwent massive apoptosis within a few hours. This event may be induced by hypoxia as well as lack of cytokines or hormones, all stresses known to induce apoptosis. However, in these conditions, the deepest cortical thymocytes did not undergo apoptosis, suggesting that medullary thymocytes are the most sensitive to stressinduced apoptosis. Our results show that the c-Rel protein became detectable, as soon as $2 \mathrm{~h}$ in culture, in the cytoplasm of most medullary thymocytes. At this stage, these thymocytes still appeared healthy, although apoptosis has already begun as evidenced by the presence of a few TUNEL-positive cells. Most thymocytes progressed into fully apoptotic cells from $3 \mathrm{~h}$ in culture and the expression of c-Rel became conspicuous. We therefore consider that after $2 \mathrm{~h}$ in culture most thymocytes were committed to die. Thus, the cRel protein would be induced in medullary thymocytes committed to die upon apoptotic stimulation. This induction would occur by a post-transcriptional mechanism since no increase in c-rel mRNA expression was detected before or during apoptosis. In conclusion, our results suggest that c-Rel is involved in naturally-occurring apoptosis of cortical thymocytes as well as in stress-induced apoptosis of medullary thymocytes.

\section{c-Rel translocates into the nucleus of thymocytes during apoptosis}

As shown above, during organotypic culture c-Rel was first detected in the cytoplasm of thymocytes committed to die. During the time course of apoptosis, the subcellular localization of c-Rel changes: from $3 \mathrm{~h}$ in culture onwards, c-Rel can be found either in the cytoplasm or in both the cytoplasm and the nucleus of thymocytes still healthy in appearance or already condensed. This change in subcellular localization was confirmed by Western blot experiments. Therefore, c-Rel seems to translocate from the cytoplasm into the nucleus of thymocytes during apoptosis. Once in the nucleus, c-Rel might modulate the transcription of some target genes involved in the mechanism of apoptosis. However, the transcriptional activity of $\mathrm{c}$-Rel during thymocyte apoptosis remains to be established and target genes have to be identified.

\section{Mechanism triggering the c-Rel nuclear translocation}

In many cell types, Rel/NF- $\kappa$ B proteins are expressed in an inactive form sequestered in the cytoplasm by an $I_{\kappa} B$ protein. 

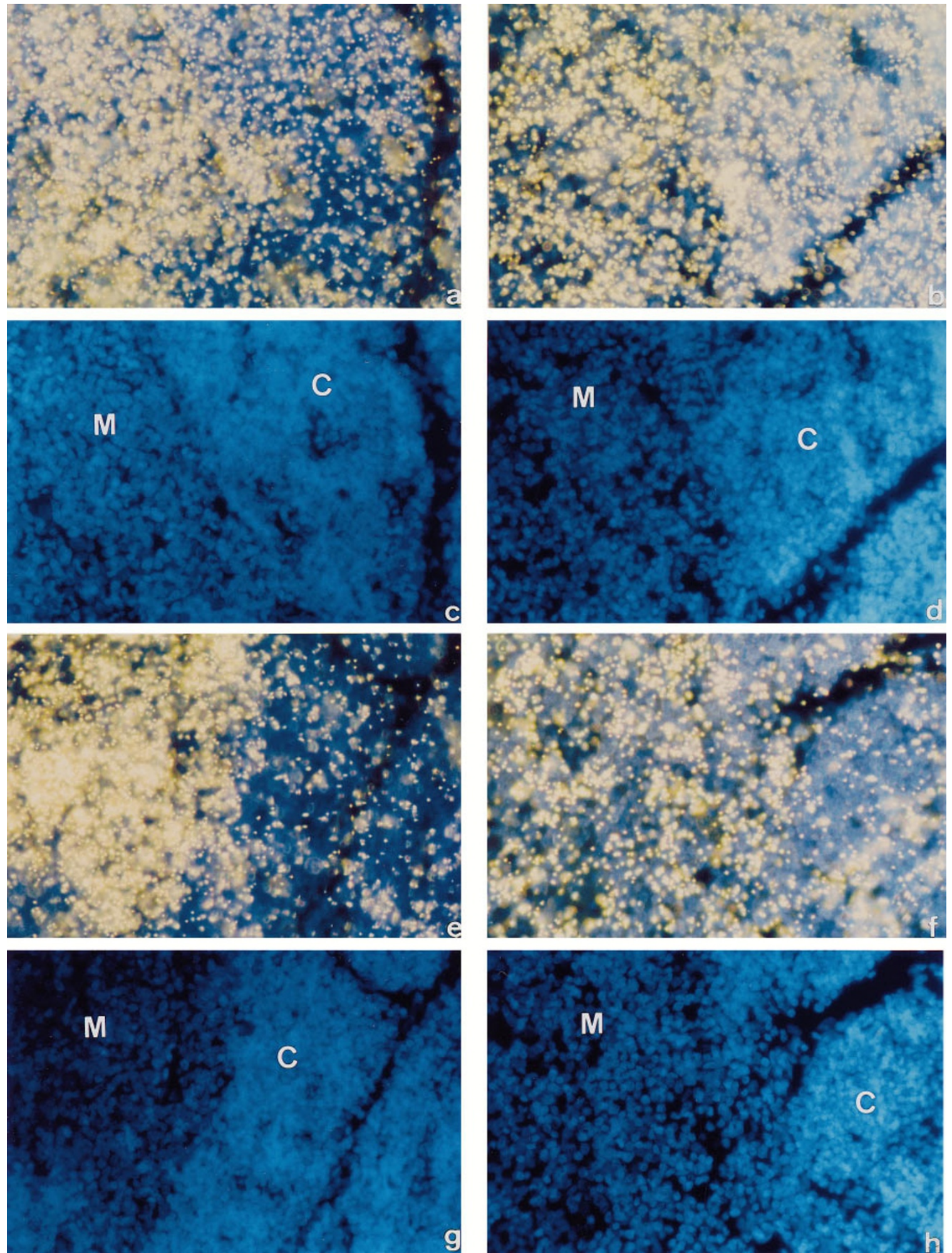

Figure 6 Detection of $c$-rel and ikba gene expression during experimentally-induced apoptosis. Sections of P1 thymuses maintained in organ culture were processed for in situ hybridization and counterstained with a fluorescent DNA intercalant. Sections are observed in dark field and UV illumination (a, b, e and f) or under UV illumination alone (c, $\mathbf{d}, \mathbf{g}$ and $\mathbf{h}$ ). In control thymus, c-rel is expressed both in the cortex (C) and the medulla (M) with a higher expression in the latter ( $\mathbf{a}$ and $\mathbf{c})$. After $2 \mathrm{~h}$ in culture, this pattern of expression does not change (b and $\mathbf{d})$. In control thymus, ikba is highly expressed in all medullary cell types and at lower level in cortical cells (e and $\mathbf{g}$ ). After $2 \mathrm{~h}$ of culture, ikba expression decreases in medullary thymocytes. The remaining expression is mainly in stromal cells. 
The major pathway allowing the activation of Rel/NF- $\kappa \mathrm{B}$ proteins involves the phosphorylation of $\mathrm{I} \kappa \mathrm{B} \alpha$ and its subsequent ubiquitination and degradation by the proteasome (review in Verma et al, 1995). Our results show that there is no detectable preexisting c-Rel proteins in medullary thymocytes before the apoptotic stimulation, indicating that the molecules which enter the nucleus are neo-synthesized. Moreover, we have previously shown that the ikba gene is highly expressed in medullary thymocytes of control thymuses (Huguet et al, 1997). Therefore, to investigate the mechanism of avian c-Rel nuclear translocation in apoptotic thymocytes, we examined ikba gene expression. From $2 \mathrm{~h}$ in culture, i.e. before the beginning of the nuclear translocation of c-Rel, ikba expression decreases. Thus, the c-Rel protein would not be sequestered as its synthesis proceed and could be able to enter the nucleus. Although the ikba gene expression was shown to be upregulated by overexpressed c-Rel in avian fibroblasts (Schatzle et al, 1995), we did not observe such an upregulation in medullary thymocytes from $3 \mathrm{~h}$ of culture. These results are the first example of an activation of c-Rel that would occur through a decrease in the transcriptional activity of $i k b a$. However, whether this decrease is alone sufficient for a complete c-Rel activation or whether it occurs together with an active degradation of the $I_{\kappa} \mathrm{B} \alpha$ protein remains to be established.

\section{Involvement of different Rel/NF- $\kappa$ B members in apoptosis}

The involvement of the avian c-Rel protein in apoptosis was previously shown for a variety of non-lymphoid cells of the avian embryo as well as for bone marrow cells (Abbadie et al, 1993). Here, we present evidence for a role of c-Rel in the apoptosis of cortical thymocytes occurring in vivo during selection as well as in stress-induced apoptosis of medullary thymocytes. Other Rel/NF- $\kappa \mathrm{B}$ members were shown to be able to induce or repress apoptosis. In a mouse T-cell hybridoma, overexpression of $\mathrm{Bcl}-2$ blocked dexamethasone-induced apoptosis and led to a decrease in RelA/p50 heterodimers and an increase in p50/p50 homodimers by a post-transcriptional mechanism (Ivanov et al, 1995). Likewise, disruption of the mouse RelA gene induced severe hepatocyte apoptosis (Beg et al, 1995). Treatment with TNF- $\alpha$ of embryonic fibroblasts and macrophages derived from these RelA-deficient mice resulted in a dramatic decrease in viability, thus suggesting that RelA protects cells from apoptosis (Beg and Baltimore, 1996). Similar conclusions were drawn from studies using human fibrosarcoma cells, human primary fibroblasts and human Jurkat T-cells overexpressing a super-repressor form of $\mathrm{I}_{\kappa} \mathrm{B} \alpha$. When treated by TNF- $\alpha$, these cells were more susceptible to apoptosis than control cells (Wang et al, 1996, Van Antwerp et al, 1996). However, in human 293 kidney cells, overexpression of a dominant-negative RelA protein inhibited apoptosis suggesting that in that case RelA induces cell death (Grimm et al, 1996). Finally, one experiment done with Hela cells has evidenced the ability of c-Rel to protect against TNF-induced apoptosis, even with a more efficiency than RelA (Liu et al, 1996). Taken together, these conflicting results illustrate the possibility that different Rel/NF- $\kappa$ B members may be activated upon apoptotic stimulation resulting in stimulation or inhibition of apoptosis depending on cell type or species.

\section{Materials and Methods}

\section{Animals}

Fertilized white Leghorn chicken eggs were incubated at $39^{\circ} \mathrm{C}$ in a humidified air chamber. The age of embryos is indicated as E1, E2..., E1 corresponding to $24 \mathrm{~h}$ of egg incubation and as P1 corresponding to $24 \mathrm{~h}$ post-hatching.

\section{Histological sections}

E9.5, E15, E19 and P1 chick embryo thymuses were dissected in PBS and fixed at $4{ }^{\circ} \mathrm{C}$ for $18 \mathrm{~h}$ in $4 \%$ paraformaldehyde in PBS $\left(\mathrm{Na}_{2} \mathrm{HPO}_{4}\right.$ -

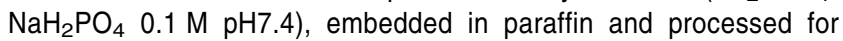
histological sections.

\section{Thymus organ culture}

E19 and P1 thymuses were rapidly dissected in culture medium (RPMI $1640,10 \%$ FCS, $2 \%$ chicken serum, $1000 \mathrm{IU} \mathrm{ml}^{-1}$ penicillin, $1000 \mu \mathrm{g} \mathrm{ml}^{-1}$ streptomycin, $2 \mathrm{mM}$ glutamine) on ice. Organs were set to float on top of millicell chambers in 6 well culture plates (Millipore) filled up with culture medium. The culture was carried out from 1 to $24 \mathrm{~h}$, at $40^{\circ} \mathrm{C}$, in $10 \% \mathrm{CO}_{2}, 95 \%$ humidity.

\section{In situ hybridization}

(a) Synthesis of ${ }^{35} \mathrm{~S}$ RNA probes: The chicken full length c-rel cDNA was cloned in Bluescript $\mathrm{SK}^{-}$(Stratagene) as described in (Abbadie et al, 1993). A $0.9 \mathrm{~kb} \mathrm{EcoRl}$ fragment from the chicken $i k b a$ cDNA, kindly provided by $\mathrm{H}$. Bose, was cloned in Bluescript $\mathrm{SK}^{-}$phagemid (Stratagene). ${ }^{35} \mathrm{~S}$ RNA probes were transcribed from $2 \mu \mathrm{g}$ of linearized plasmids by $20 \mathrm{U}$ of either T7, T3 or SP6 RNA polymerase for sense and antisense probes in a $20 \mu \mathrm{l}$ reaction mixture containing $20 \mu \mathrm{Ci}^{35} \mathrm{~S}-\mathrm{CTP}(1300 \mathrm{Ci} / \mathrm{mmole}), 200 \mu \mathrm{M}$ UTP, ATP and GTP for $1 \mathrm{~h}$ at $39^{\circ} \mathrm{C}$. To facilitate their penetration into cells, probes were submitted to a limited alkaline hydrolysis generating fragments of approximately 150 bases as recommended by Cox et al, (1984). (b) In situ hybridization: In situ hybridization was adapted from the method of Cox et al (1984), as described by Quéva et al (1992). Briefly, after being deparaffinized and rehydrated, sections were incubated in $0.1 \mathrm{M}$ glycine, $0.2 \mathrm{M}$ Tris$\mathrm{HCl} \mathrm{pH} 7.4$ for $10 \mathrm{~min}$ at $20^{\circ} \mathrm{C}$, treated with $1 \mu \mathrm{g} \mathrm{ml}^{-1}$ proteinase $\mathrm{K}$ (Boehringer Mannheim) for $15 \mathrm{~min}$ at $37^{\circ} \mathrm{C}$, post-fixed in $4 \%$ paraformaldehyde, washed in PBS, acetylated $10 \mathrm{~min}$ with $0.25 \%$ acetic anhydride in $0.1 \mathrm{M}$ triethanolamine, washed in $2 \times$ SSC $(0.3 \mathrm{M}$ $\mathrm{NaCl}, 30 \mathrm{mM}$ sodium citrate) and dehydrated in ethanol. ${ }^{35} \mathrm{~S}$ RNA probes were denatured at $80^{\circ} \mathrm{C}$ and diluted in the hybridization buffer at a concentration of $50 \mathrm{pg} \mu \mathrm{l}^{-1}$. Hybridization was performed at $60^{\circ} \mathrm{C}$ for $18 \mathrm{~h}$. After a wash in $4 \times \mathrm{SSC}$ at $20^{\circ} \mathrm{C}$, slides were treated with $10 \mu \mathrm{g} \mathrm{ml}^{-1}$ of RNase A (type III A, Sigma) for $30 \mathrm{~min}$ at $37^{\circ} \mathrm{C}$, subsequently washed in $0.1 \times \mathrm{SSC}$ at $60^{\circ} \mathrm{C}$, dehydrated by ethanol and dipped in nuclear track emulsion (Kodak NTB2). Slides were exposed at $4^{\circ} \mathrm{C}$ for 2 weeks. After developing, sections were stained with a DNA intercalating fluorescent dye (Hoechst 33258), mounted and observed under dark-field and UV illumination with a Zeiss microscope. Both antisense and sense probes were used and the sense probes never gave any signal. 


\section{Immunocytochemistry and Western-blot}

(a) Antibodies: A rabbit immuno-purified anti-c-Rel serum (SB146) raised against the 15 carboxy terminal amino acids specific of the chicken c-Rel protein (Abbadie et al, 1993) was used both in Western-blot and immunocytochemistry experiments. Normal rabbit immunoglobulin fraction (Dako) was used as negative control. (b) Immunocytochemistry: After being deparaffinized and rehydrated, sections were incubated in $0.5 \mathrm{mg} \mathrm{ml}^{-1}$ saponine (Sigma) in PBS for $30 \mathrm{~min}$; then endogenous peroxidase activity was quenched by incubation in $80 \%$ methanol, $20 \% \mathrm{PBS}, 0.6 \% \mathrm{H}_{2} \mathrm{O}_{2}$ for $30 \mathrm{~min}$ followed by $30 \mathrm{~min}$ saturation in $5 \%$ milk in PBS. Sections were incubated with the primary antibody overnight at $4^{\circ} \mathrm{C}$. After three washes in PBS, the EXTRA-3 kit (Sigma Immunochemicals) and the AEC substrate system (Dako) were used to detect the primary antibody. Sections were counterstained with hematoxylin (Sigma) and mounted in Glycergel (Dako). (c) Western blot of cytoplasmic and nuclear extracts: To separate nuclear from cytoplasmic proteins, cultured thymuses were collected in a solution containing $0.015 \mathrm{M}$ Hepes $\mathrm{pH} 7.9,0.014 \mathrm{M} \quad \beta$-mercaptoethanol, $0.3 \mathrm{M}$ Saccharose, $0.060 \mathrm{M} \mathrm{KCl}, 0.015 \mathrm{M} \mathrm{NaCl}, 0.15 \mathrm{mM}$ spermine, $0.5 \mathrm{mM}$ spermidine, $0.5 \mathrm{mM}$ EGTA, $0.002 \mathrm{M}$ EDTA. After suspension in a Dounce homogenizer on ice, $3 \%$ Nonidet P40 was added and samples incubated for $5 \mathrm{~min}$ on ice, then centrifuged $2 \mathrm{~min}$ at $10,000 \mathrm{~g}$ at $4{ }^{\circ} \mathrm{C}$. The supernatants containing cytoplasmic proteins were stored in aliquots at $-80^{\circ} \mathrm{C}$. The pellets of nuclei were resuspended in nine volumes of NUN $1.1 \times$ buffer $(1.1 \mathrm{M}$ Urea, $0.33 \mathrm{M} \mathrm{NaCl}, 1.1 \%$ Nonidet P40, $0.27 \mathrm{M}$ Hepes $\mathrm{pH} 7.6$ ), incubated for $15 \mathrm{~min}$ on ice, and centrifuged $15 \mathrm{~min}$ at $10,000 \mathrm{~g}$ at $4^{\circ} \mathrm{C}$ (Schibler et al, 1993; Lavery and Schibler, 1993). The supernatants containing nuclear proteins were stored in aliquots at $-80^{\circ} \mathrm{C}$. Protein concentration was measured by optic density at $260 \mathrm{~nm}$. Equiloaded $10 \%$ SDS-polyacrylamide gels were run and blotted onto nitrocellulose sheets. Nitrocellulose sheets were saturated $1 \mathrm{~h}$ with $5 \%$ milk in PBS and then incubated overnight at $4{ }^{\circ} \mathrm{C}$ with SB146. After three washes in PBS, the sheets were incubated with an anti-rabbit antibody for $2 \mathrm{~h}$ at room temperature. After washing, the peroxidase activity was revealed by incubation with a chemiluminescent substrate (Enhanced Chemiluminescence system, Amersham).

\section{TUNEL staining}

The TUNEL protocol was adapted from the Apoptag kit instructions (APOPTAG ${ }^{(i)}$ PLUS, Oncor). Briefly, after being deparaffinized and rehydrated, sections were incubated in $0.1 \mathrm{M}$ glycine, $0.2 \mathrm{M}$ Tris buffer $\mathrm{pH} 7.4$ for $10 \mathrm{~min}$ at room temperature, then digested by $20 \mu \mathrm{g} \mathrm{ml}^{-1}$ proteinase $\mathrm{K}$ in $0.05 \mathrm{M}$ EDTA, $0.1 \mathrm{M}$ Tris buffer $\mathrm{pH} 8$ for $15 \mathrm{~min}$ at $37^{\circ} \mathrm{C}$ and finally incubated in $80 \%$ methanol, $20 \% \mathrm{PBS}$, $0.6 \% \mathrm{H}_{2} \mathrm{O}_{2}$ for $30 \mathrm{~min}$ to quench endogenous peroxidase activity. The following steps were carried out as described in the kit. Sections were finally counterstained with hematoxylin and mounted in Glycergel (Dako).

\section{Acknowledgements}

We are grateful to Eric Maire and Serge Plazza for their technical help and advice. This work was supported by grants from the Institut Pasteur de Lille, the Centre National de la Recherche Scientifique, the Association pour la Recherche sur le Cancer and the Groupement des Enreprises Françaises pour la Lutte contre le Cancer. C.H. was supported by a training grant from the Association pour la Recherche sur le Cancer.

\section{References}

Abbadie C, Kabrun N, Bouali F, Smardova J, Stéhelin D, Vandenbunder B and Enrietto $P$ (1993) High levels of c-rel expression are associated with programmed cell death in the developing avian embryo and in bone marrow cells in vitro. Cell 75: $899-912$

Bauerle PA and Henkel T (1994) Function and activation of NF- $\kappa \mathrm{B}$ in the immune system. Annu. Rev. Immunol 12: 141-179

Beg AA and Baltimore D (1996) An essential role for NF- $\kappa$ B in preventing TNF- $\alpha$ induced cell death. Science 274: $782-784$

Beg AA, Sha WC, Bronson RT, Ghosh S and Baltimore D (1995) Embryonic lethality and liver degeneration in mice lacking the RelA component of NF- $\kappa$ B. Nature 376 : $167-170$

Capobianco AJ, Simmons DL and Gilmore TD (1990) Cloning and expression of a chicken c-relcDNA: unlike p59 ${ }^{\mathrm{v}-\mathrm{rel}}, \mathrm{p} 68^{\mathrm{c}-\mathrm{rel}}$ is a cytoplasmic protein in chicken embryo fibroblasts. Oncogene 5: 257-265

Carrasco D, Weih F and Bravo R (1994) Developmental expression of the mouse $c$ rel proto-oncogene in hematopoietic organs. Development 120: 2991-3004

Cox KH, DeLeon DV, Angerer LM and Angerer RC (1984) Detection of mRNAs in sea urchin embryos by in situ hybridization using asymmetric RNA probes. Dev. Biol. 101: $485-502$

Davidson NJ, Chen CIH and Boyd RL (1992) Kinetics of chicken embryonic thymocyte development in ovo and in organ culture. Eur. J. Immunol. 22: 1429 1435

Davis N, Ghosh S, Simmons DL, TempstP, Liou HC, Baltimore D and Bose HJ (1991) Rel-associated pp40: an inhibitor of the Rel family of transcription factors. Science 253: 1268-1271

Diehl JA, MacKinsey TA and Hannink M (1993) Differential pp40/l $\kappa$ B- $\beta$ inhibition of DNA binding by Rel proteins. Mol. Cell. Biol. 13: 1769-1778

Egerton M, Scollay Rand Shortman K (1990) Kinetics of mature T-cell development in the thymus. Proc. Natl. Acad. Sci. USA 87: 2579-2582

Gavrieli Y, Sherman Y and Ben-Sasson SA (1992) Identification of programmed cell death in situ via specific labeling of nuclear DNA fragmentation. J. Cell Biol. 119: 493-501

Gilmore TD, Koedood M, Piffat KA and White DW (1996) Rel/NF- $\kappa$ B// $\kappa$ B proteins and cancer. Oncogene 13: 1367-1378

Grimm S, Bauer MKA, Baeuerle PA and Schulze-Osthoff K (1996) Bcl-2 downregulates the activity of transcription factor NF- $\kappa$ B induced upon apoptosis. J. Cell Biol. 134: 13-23

Huguet C, Enrietto PJ, Vandenbunder B and Abbadie C (1994) c-Rel: a multifunctional transcription factor? Cell Death Differ. 1: 71-76

Ivanov VN, Deng G, Podack ER and Malek TR (1995) Pleiotropic effects of Bcl-2 on transcription factors in T cells: potential role of NF- $\kappa B$ p50-p50 for the antiapoptotic function of Bcl-2. International Immunology 7: 1709-1720

Kerr LD, Inoue J, Davis N, LinkE, Baeuerle PA, Bose HJ and Verma IM (1991) The relassociated pp40 protein prevents DNA binding of Rel and NF-kappa B: relationship with I kappa B beta and regulation by phosphorylation. Genes and Development 5: $1464-1476$

Köntgen F, Grumont RJ, Strasser A, Metcalf D. Li R, Tarlinton D and Gerondakis S (1995) Mice lacking the c-rel proto-oncogene exhibit defects in lymphocyte proliferation, humoral immunity, and interleukin-2 expression. Genes and Development 9: 1965-1977

Lavery DJ and Schibler U (1993) Circadian transcription of the cholesterol 7a hydroxylase gene may involve the liver-enriched bZIP protein DBP. Genes and Development 7: 1871-1884

Liu Z, Hsu H, Goeddel DV and Karin M (1996) Dissection of TNF receptor 1 effector functions: JNK activation is not linked to apoptosis while NF- $\kappa$ B activation prevents cell death. Cell 87: $565-576$

Miyamoto $S$ and Verma IM (1995) Rel/NF- $\kappa$ B/l $\kappa$ B story. Adv. Cancer Res. 66: $255-$ 292

Moore BE and Bose HR (1989) Expression of the c-reland c-myc proto-oncogenes in avian tissues. Oncogene 4: 845-852

Neiman PE, Thomas SJ and Loring G (1991) Induction of apoptosis during normal and neoplastic B-cell development in the bursa of Fabricius. Proc. Natl. Acad. Sci. USA 88: $5857-5861$

Nossal GJV (1994) Negative selection of lymphocytes. Cell 76: 229-239

Quéva C, Ness SA, Graf T, Vandenbunder B and Stéhelin D (1992) Expression patterns of $c$-myb and of $c$-myb induced myeloid-1 (mim-1) gene during the development of the chick embryo. Development 114: 125-133 
Schatzle JD, Kralova J and Bose HR (1995) Avian $\left.\right|_{\kappa} B \alpha$ is transcriptionally induced by c-Rel and v-Rel with different kinetics. Journal of Virology 69: 5383-5390

Schibler U, Hagenbüchle O, Wellauer PK and Pittet AC (1983) Two promoters of different strengths control the transcription of the mouse alpha-amylase gene Amy-1a in the parotid gland and the liver. Cell 33: 501-508

Van Antwerp DJ, Martin SJ, Kafri T, Green DR and Verma IM (1996) Suppression of TNF- $\alpha$-induced apoptosis by NF- $\kappa$ B. Science 274: 787-789

Verma IM, Stevenson JK, Schwarz EM, Van Antwerp D and Miyamoto S (1995) Rel/ NF- $\kappa$ B/l $\kappa$ B family: intimate tales of association and dissociation. Genes and Development 9: 2723-2735 von Boehmer H (1994) Positive selection of lymphocytes. Cell 76: 219-228

Wang C, Mayo MW and Baldwin ASJ (1996) TNF- and cancer therapy-induced apoptosis: potentiation by inhibition of NF- $\kappa$ B. Science $274: 784-787$

White DW and Gilmore TD (1996) Bcl-2 and CrmA have different effects on transformation, apoptosis and the stability of $I_{\kappa} \mathrm{B} \alpha$ in chicken spleen cells transformed by temperature-sensitive v-Rel oncoproteins. Oncogene 13: 891 899

White DW, Roy A and Gilmore TD (1995) The v-Rel oncoprotein blocks apoptosis and proteolysis of $\mid \kappa B$ - $\alpha$ in transformed chicken spleen cells. Oncogene 10:857-868 